\title{
GENERALIZED LEAST-SQUARES SOLUTIONS TO QUASI-LINEAR INVERSE PROBLEMS WITH A PRIORI INFORMATION
}

\author{
Mitsuhiro MAtsu'Ura and Naoshi HrRata \\ Geophysical Institute, Faculty of Science, \\ the University of Tokyo, Tokyo, Japan \\ (Received July 19, 1982; December 27, 1982)
}

\begin{abstract}
A quasi-linear inverse problem with a priori information about model parameters is formulated in a stochastic framework by using a singular value decomposition technique for arbitrary rectangular matrices.

In many geophysical inverse problems, we have a priori information from which a most plausible solution and the statistics of its probable error can be guessed. Starting from the most plausible solution, the optimization of model parameters is made by the successive iteration of solving a set of standardized linear equations for corrections at each step. In under-determined cases, the solution depends inherently on the initial guess of model parameters, and then uncertainties in the solution are evaluated by the covariances of estimation error which results not only from the random noise in data but also from the probable error in the initial guess.

A best linear inverse operator which minimizes the variances of estimation errors within the framework of a generalized least-squares approach is directly obtained from the "natural inverse" of Lanczos for a coefficient matrix by regarding an eigenvalue in the inverse as zero if it is smaller than unity. This provides a theoretical basis on the "sharp cutoff approach" of Wiggins and also Jackson in their general inverse formalisms.
\end{abstract}

\section{Introduction}

Many problems in geophysics involve estimating unknown parameters from non-linear observation equations with random noise. These problems are usually solved by repeating successively the linearization of observation equations in the neighborhood of a most plausible estimate and the solution of a set of linear equations for corrections.

BACKUS and GrLBERT $(1967,1968,1970)$ have published a series of papers dealing with the geophysical inverse problem, in which they made detailed investigations of trade-off between resolution and error in the estimation of unknown variables, and constructed a general inverse formalism in the context of determining the earth's structure from free oscillation observations. General treatments of the linear inverse problem have been developed by JACKSON (1972) and WIGGINS (1972) in terms of a simple matrix algebra. In their formalism, the inverse oper- 
ator which optimizes the trade-off between resolution and estimation error is directly constructed from the "natural inverse" of LANCZOS (1961) for a coefficient matrix by truncating the smallest eigenvalues in the inverse so that the estimation error remains less than the certain maximum error determined a priori from requirement for definiteness in the solution.

On the other hand, FRANKLIN (1970) treated the inverse problem in a stochastic framework, and found the best linear estimator which minimizes the statistical average of the discrepancy between true solution and estimate. His result includes the damped least-squares solution by LEVENBERG (1944) and MARQUARDT $(1963,1970)$ as a special case. Recently, JACKSON (1979) showed that an ill-posed linear system can be always reduced to a well-posed linear system by incorporating sufficient a priori data into observed data, and that the problem is solved by using the usual least-squares procedure. Jackson's approach yields the solution which minimizes the total variances involving both resolving error and data error. In a special case, this solution becomes formally identical with the Franklin's best linear estimate.

The purpose of the present paper is to construct a general formalism for a quasi-linear inverse problem with a priori information by using a singular value decomposition technique for arbitrary rectangular matrices and to clarify the relationships between our approach and those of the previous works listed above.

\section{Singular Value Decomposition for Rectangular Matrices}

According to Lanczos (1961), an arbitrary $n \times m$ matrix $\boldsymbol{A}$ of rank $p(<n, m)$ can be decomposed into the product of an orthonormal $n \times n$ matrix $U$, a semidiagonal $n \times m$ matrix $\Lambda$ and a transposed orthonormal $m \times m$ matrix $\tilde{V}$, that is

$$
A=U \Lambda \tilde{V}=\left[U_{p} \mid U_{0}\right]\left[\begin{array}{l|l}
\Lambda_{p} & O \\
\hline O & O
\end{array}\right]\left[\begin{array}{l}
\tilde{V}_{p} \\
\hline \tilde{V}_{0}
\end{array}\right]=U_{p} \Lambda_{p} \tilde{V}_{p}
$$

with

$$
\left.\begin{array}{ll}
\boldsymbol{U}_{p}=\left[\boldsymbol{u}_{1}, \cdots, \boldsymbol{u}_{p}\right], & \boldsymbol{U}_{0}=\left[\boldsymbol{u}_{p+1}, \cdots, \boldsymbol{u}_{n}\right], \\
\boldsymbol{V}_{p}=\left[\boldsymbol{v}_{1}, \cdots, \boldsymbol{v}_{p}\right], & \boldsymbol{V}_{0}=\left[\boldsymbol{v}_{p+1}, \cdots, \boldsymbol{v}_{m}\right],
\end{array}\right\}
$$

and

$$
\boldsymbol{\Lambda}_{p}=\left[\begin{array}{cc}
\lambda_{1} & 0 \\
\ddots & \ddots \\
0 & \lambda_{p}
\end{array}\right], \quad \lambda_{1} \geq \lambda_{2} \geq \cdots \geq \lambda_{p}>0
$$

Here, the $n$-dimensional column vector $\boldsymbol{u}_{i}$, the $m$-dimensional column vector $\boldsymbol{v}_{i}$, and the non-zero diagonal elements $\lambda_{i}$ are defined by the "coupled eigenvalue equations,"

$$
\left.\begin{array}{c}
A v_{i}=\lambda_{i} u_{i} \\
\tilde{A} u_{i}=\lambda_{i} v_{i}
\end{array}\right\} \quad(i=1, \cdots, p)
$$

for positive eigenvalues, and the "decoupled eigenvalue equations," 


$$
\left.\begin{array}{ll}
A \boldsymbol{v}_{i}=0 & (i=p+1, \cdots, m) \\
\tilde{A u}_{i}=0 & (i=p+1, \cdots, n),
\end{array}\right\}
$$

for zero eigenvalues. The above factorization of matrix is known as a singular value decomposition, and it was developed by ECKART and Young (1939) for general matrices.

From the orthogonality of the matrices $U$ and $V$, we obtain the following relations; for $U_{p}$ and $U_{0}$,

$$
\left.\begin{array}{l}
\tilde{U}_{p} \boldsymbol{U}_{p}=\boldsymbol{I}_{p}, \quad \tilde{U}_{0} \boldsymbol{U}_{0}=\boldsymbol{I}_{n-p}, \quad \boldsymbol{U}_{p} \tilde{U}_{p}+\boldsymbol{U}_{0} \tilde{U}_{0}=\boldsymbol{I}_{n}, \\
\tilde{U}_{0} \boldsymbol{U}_{p}=\boldsymbol{O}_{(n-p) \times p}, \quad \tilde{U}_{p} \boldsymbol{U}_{0}=\boldsymbol{O}_{p \times(n-p)},
\end{array}\right\}
$$

and for $V_{p}$ and $V_{0}$,

$$
\left.\begin{array}{l}
\tilde{V}_{p} V_{p}=I_{p}, \quad \tilde{V}_{0} V_{0}=I_{m-p}, \quad V_{p} \tilde{V}_{p}+V_{0} \tilde{V}_{0}=I_{m}, \\
\tilde{V}_{0} V_{p}=\boldsymbol{O}_{(m-p) \times p}, \quad \tilde{V}_{p} V_{0}=\boldsymbol{O}_{p \times(m-p)},
\end{array}\right\}
$$

where $\boldsymbol{I}_{k}$ and $\boldsymbol{O}_{k \times l}$ denote a $k \times k$ unit matrix and a $k \times l$ null matrix, respectively.

\section{Analysis of a Linear System}

First of all, we analyze an arbitrary linear system without regard for random noise in the data, and try to find a best solution with the generalized least-squares.

In matrix notation, a set of $n$ linear equations with $m$ unknown parameters is written as:

$$
\boldsymbol{y}=\boldsymbol{A x},
$$

where $\boldsymbol{y}$ is an $n$-dimensional data vector, $\boldsymbol{x}$ is an $m$-dimensional solution vector, and $\boldsymbol{A}$ is an $n \times m$ coefficient matrix. Denoting the rank of the coefficient matrix $\boldsymbol{A}$ by $p(<n, m)$, the singular value decomposition of $\boldsymbol{A}$ is given in the same form as in Eq. (2.1). Transformations of $\boldsymbol{y}, \boldsymbol{x}$, and $\boldsymbol{A}$ by the orthonormal matrices $\boldsymbol{U}$ and $V$;

$$
\begin{aligned}
& y^{\prime}=\tilde{U} y=\left[\frac{\tilde{U}_{p} y}{\tilde{U}_{0} y}\right], \quad x^{\prime}=\tilde{V} x=\left[\frac{\tilde{V}_{p} x}{\tilde{V}_{0} x}\right], \\
& A^{\prime}=\tilde{U} A V=\Lambda=\left[\frac{\Lambda_{p}}{O} \mid \frac{O}{O}\right],
\end{aligned}
$$

yield a new linear system equivalent to Eq. (3.1) as

$$
\boldsymbol{y}^{\prime}=\boldsymbol{\Lambda} \boldsymbol{x}^{\prime},
$$

or, denoting an arbitrary column vector of $m-p$ components by $\eta$,

with

$$
\left.\begin{array}{l}
y_{p}{ }^{\prime}=\Lambda_{p} x_{p}{ }^{\prime}, \\
y_{0}{ }^{\prime}=0, \quad x_{0}{ }^{\prime}=\eta
\end{array}\right\}
$$

$$
\left.\begin{array}{ll}
\boldsymbol{y}_{p}{ }^{\prime}=\tilde{U}_{p} y, & y_{0}{ }^{\prime}=\tilde{U}_{0} y, \\
x_{p}{ }^{\prime}=\tilde{V}_{p} x, & x_{0}{ }^{\prime}=\tilde{V}_{0} x
\end{array}\right\}
$$


The above equations show that the linear system has a unique and complete solution, if the system is well-posed $(m=n=p)$. In other cases, the linear system has no unique and complete solution, but there still exists a unique solution in terms of the generalized least-squares.

From the inverse transformation of Eq. (3.2), we obtain

$$
\left.\begin{array}{l}
\boldsymbol{y}=\boldsymbol{U} \boldsymbol{y}^{\prime}=U_{p} y_{p}{ }^{\prime}+U_{0} y_{0}{ }^{\prime}, \\
\boldsymbol{x}=\boldsymbol{V} \boldsymbol{x}^{\prime}=V_{p} \boldsymbol{x}_{p}{ }^{\prime}+V_{0} \boldsymbol{x}_{0}{ }^{\prime} .
\end{array}\right\}
$$

By using the above relations, the residual vector, $\boldsymbol{r} \equiv \boldsymbol{y}-\boldsymbol{A x}$, can be expressed in the transformed notation as

$$
\boldsymbol{r} \equiv \boldsymbol{y}-\boldsymbol{A} \boldsymbol{x}=\boldsymbol{U}_{p}\left(\boldsymbol{y}_{p}{ }^{\prime}-\Lambda_{p} \boldsymbol{x}_{p}{ }^{\prime}\right)+\boldsymbol{U}_{0} \boldsymbol{y}_{0}{ }^{\prime},
$$

and the square of the Euclidean norm of the residual vector, $\|\boldsymbol{r}\|^{2}=\tilde{\boldsymbol{r}} \boldsymbol{r}$, is given by

$$
\|\boldsymbol{r}\|^{2}=\|\boldsymbol{y}-A \boldsymbol{x}\|^{2}=\left\|\boldsymbol{y}_{p}{ }^{\prime}-\Lambda_{p} \boldsymbol{x}_{p}{ }^{\prime}\right\|^{2}+\left\|\boldsymbol{y}_{0}{ }^{\prime}\right\|^{2} .
$$

The least-squares solution, which minimizes $\|\boldsymbol{r}\|^{2}$, requires

$$
\boldsymbol{x}_{p}{ }^{\prime}=\boldsymbol{\Lambda}_{p}{ }^{-1} \boldsymbol{y}_{p}{ }^{\prime}
$$

leaving $x_{0}{ }^{\prime}$ free. From Eqs. (3.5), (3.6), and (3.9), we obtain a general expression of the least-squares solution in the original notation as follows;

$$
\boldsymbol{x}=V_{p} \Lambda_{p}{ }^{-1} \tilde{U}_{p} \boldsymbol{y}+\boldsymbol{V}_{0} \eta
$$

where $\eta$ is an arbitrary column vector of $m-p$ components.

The above expression shows that the least-squares solution is unique if $p=m$. When $p<m$, the linear system in Eq. (3.1) has an infinite number of solutions which satisfy the least-squares criterion. However, even in such a case, we can define a unique solution $\hat{\boldsymbol{x}}$ which minimizes the square of the Euclidean norm of the solution vector,

by putting $\eta=0$ in Eq. (3.10), i.e.,

$$
\|\boldsymbol{x}\|^{2}=\left\|\boldsymbol{\Lambda}_{p}^{-1} \tilde{U}_{p} \boldsymbol{y}\right\|^{2}+\|\eta\|^{2},
$$

$$
\hat{\boldsymbol{x}}=A^{\dagger} \boldsymbol{y}
$$

with

$$
A^{\dagger}=\boldsymbol{V}_{p} \boldsymbol{\Lambda}_{p}^{-1} \tilde{U}_{p}
$$

The inverse operator $A^{\dagger}$ was introduced by LANCzos (1961) as the "natural inverse" of matrix $A$, which is equivalent to the "generalized inverse" of PENROSE (1955).

\section{Linearization and Standardization of the Problem}

We consider a set of observed data $Y_{i}(i=1, \cdots, n)$ with random noise $N_{i}$ $(i=1, \cdots, n)$ which is related to a set of unknown model parameters $X_{j}(j=1, \cdots$, $m)$ by quasi-linear functions $F_{i}(i=1, \cdots, n)$ : that is, in vector notation, 


$$
Y=F(X)+N
$$

with

$$
\boldsymbol{X}=\left[\begin{array}{c}
X_{1} \\
\vdots \\
\dot{X}_{m}
\end{array}\right], \quad \boldsymbol{Y}=\left[\begin{array}{c}
Y_{1} \\
\vdots \\
\dot{Y}_{n}
\end{array}\right], \quad \boldsymbol{N}=\left[\begin{array}{c}
N_{1} \\
\vdots \\
\dot{N}_{n}
\end{array}\right]
$$

and

$$
\boldsymbol{F}(\boldsymbol{X})=\left[\begin{array}{c}
F_{1}(X) \\
\vdots \\
F_{n}(X)
\end{array}\right] .
$$

Here, the random noise, which results from both inaccurate measurement and insufficient modeling, is unknown in itself, but its statistics are usually known.

Our problem is to find the best solution to the above observation equation under a certain criterion. In many geophysical inverse problems, we have $a$ priori information about model parameters: in some cases, the a priori information may result from a previous inversion analysis of a different data set, and in other cases, it may be obtained from physical consideration for model parameters (e.g., HIRATA et al., 1983; MATSU'URA and IwASAKI, 1983). Then, from such a priori information, we can guess a most plausible solution $\boldsymbol{X}^{0}$ and its probable error $\boldsymbol{M}$ in a statistical sense. When the function $F(X)$ is quasi-linear, the problem can be linearized by expanding $F(X)$ at $X=X^{0}$ in a power series and discarding all the second- and higher-order terms as

$$
Y=Y^{0}+B\left(X-X^{0}\right)+N
$$

with

$$
Y^{0}=F\left(X^{0}\right), \quad B=\left.\operatorname{grad} F(X)\right|_{X=X^{0}},
$$

where $\boldsymbol{B}$ is a rectangular matrix of $n$ rows and $m$ columns.

The statistics of the probable error $M$ in the initial guess and the random noise $N$ in the data are generally described in terms of an $m \times m$ covariance matrix $C\{M\}$ and an $n \times n$ covariance matrix $C\{N\}$, respectively. Since each of these matrices is symmetric and positive definite in itself, applying the fundamental decomposition theorem, we obtain

$$
C\{M\}=W_{M} \Sigma_{M} \tilde{W}_{M}, \quad C\{N\}=W_{N} \Sigma_{N} \tilde{W}_{N},
$$

and then

$$
C^{-1}\{M\}=W_{M} \Sigma_{M}^{-1} \tilde{W}_{M}, \quad C^{-1}\{N\}=W_{N} \Sigma_{N}^{-1} \tilde{W}_{N},
$$

where $W_{M}$ and $W_{N}$ are orthogonal eigenvector matrices, and $\boldsymbol{\Sigma}_{M}$ and $\boldsymbol{\Sigma}_{N}$ are diagonal matrices whose nonzero elements are the eigenvalues of $\boldsymbol{C}\{\boldsymbol{M}\}$ and $C\{N\}$, respectively.

In general, solutions to geophysical problems are determined so as to minimize or maximize some measures of uniqueness and uncertainty. Then, the problems 
should be treated in a standardized (dimensionless and statistically independent) system. According to Kaula (1966), we define weighting matrices, $\boldsymbol{G}$ in the solution space and $E$ in the data space, by

$$
C^{-1}\{M\}=\tilde{G} G, \quad C^{-1}\{N\}=\tilde{E} E,
$$

and obtain

$$
\left.\begin{array}{ll}
G=\Sigma_{M}^{-1 / 2} \tilde{W}_{M I}, & G^{-1}=W_{M} \Sigma_{M}^{1 / 2}, \\
E=\Sigma_{N}^{-1 / 2} \tilde{W}_{N}, & E^{-1}=W_{N} \Sigma_{N}^{1 / 2} .
\end{array}\right\}
$$

By using these weighting matrices, the original linear system in Eq. (4.4) is transformed into the standard form;

$$
y=y^{0}+A\left(x-x^{0}\right)+n
$$

with

$$
\begin{aligned}
& x=G X, \quad x^{0}=G X^{0}, \quad m=G M, \\
& \left.y=E Y, \quad y^{0}=E Y^{0}, \quad n=E N,\right\}
\end{aligned}
$$

and

$$
A=E B G^{-1} \text {. }
$$

Here, it should be noted that the transformed covariance matrices, $\boldsymbol{C}\{\boldsymbol{m}\}$ and $\boldsymbol{C}\{\boldsymbol{n}\}$, become unit diagonal, i.e.,

$$
C\{\boldsymbol{m}\}=G C\{\boldsymbol{M}\} \tilde{G}=I_{m}, \quad C\{\boldsymbol{n}\}=E C\{N\} \tilde{E}=I_{n} .
$$

\section{Error Analysis in a Stochastic Framework}

In the standardized system, we obtain a matrix equation,

$$
\Delta y=A \Delta x+n
$$

with

$$
\Delta x=x-x^{0}, \quad \Delta y=y-y^{0},
$$

to be solved for the correction $\Delta \boldsymbol{x}$ to the initial guess $\boldsymbol{x}^{0}$. Letting $\boldsymbol{H}$ be a linear inverse operator to the problem, the correction vector $\Delta \hat{\boldsymbol{x}}$ is given by

$$
\Delta \hat{x}=H \Delta y,
$$

and then the solution vector $\hat{\boldsymbol{x}}$ is written as

$$
\hat{x}=x^{0}+\Delta \hat{x}=H A x+(I-H A) x^{0}+H n
$$

or, by putting $\boldsymbol{m}=\boldsymbol{x}^{0}-\boldsymbol{x}$,

$$
\hat{x}=x+(I-H A) m+H n .
$$

The above relations indicate that the estimate $\hat{x}$ depends inherently on the initial guess $\boldsymbol{x}^{0}$ except for the case of $\boldsymbol{H A}=\boldsymbol{I}$. From Eqs. (4.13) and (5.5), we can 
evaluate the covariance of estimation error $\hat{x}-x$ in the standardized system as

$$
\begin{aligned}
C\{\hat{x}\} & =(I-H A) C\{m\}(\widetilde{I-H A})+H C\{n\} \tilde{H} \\
& =(I-H A)(\widetilde{I-H A})+H \tilde{H},
\end{aligned}
$$

supposing that the probable error $\boldsymbol{m}$ in the initial guess and the random noise $\boldsymbol{n}$ in the data are uncorrelated with each other. Here, the first term of the righthand side of Eq. (5.6) is related to the resolving error resulting from the error in the initial guess, and the second term to the random error resulting from the noise in the data. A similar expression of the total covariance matrix has been obtained by JACKSON (1979) from a different point of view in the construction of a general formalism of a linear inverse problem with a priori data.

Now we take the generalized inverse $A^{\dagger}$ of the coefficient matrix $A$ as the linear inverse operator $\boldsymbol{H}$. As is shown in Sec. 3, denoting the rank of $\boldsymbol{A}$ by $p$ $(\leq n, m)$, the generalized inverse operator $A^{\dagger}$ produces the correction vector,

$$
\Delta \hat{x}_{p}=A^{\dagger} \Delta y=V_{p} \Lambda_{p}{ }^{-1} \tilde{U}_{p} \Delta y,
$$

which minimizes the Euclidean norm of the residual vector, $\|\Delta y-A \Delta x\|$. The solution vector and corresponding total covariance matrix are respectively given by

$$
\hat{\boldsymbol{x}}_{p}=\boldsymbol{R} \boldsymbol{x}+(\boldsymbol{I}-\boldsymbol{R}) \boldsymbol{x}^{0}+\boldsymbol{A}^{\dagger} \boldsymbol{n}
$$

and

$$
C\left\{\hat{\boldsymbol{x}}_{p}\right\}=(\boldsymbol{I}-\boldsymbol{R})(\widetilde{\boldsymbol{I}-\boldsymbol{R}})+\boldsymbol{A}^{\dagger} \tilde{A}^{\dagger}
$$

with

$$
R=A^{\dagger} A=V_{p} \tilde{V}_{p} .
$$

Here, $\boldsymbol{R}$ is the resolution matrix introduced by WIGGINS (1972). It is noticed that the resolution matrix becomes unit diagonal if and only if $p=m$, which corresponds to the case of the classical least-squares solution.

By using the relations among the semi-orthogonal eigenvector matrices, $\boldsymbol{U}_{p}, \boldsymbol{U}_{0}, \boldsymbol{V}_{p}$ and $\boldsymbol{V}_{0}$, in Eqs. (2.6) and (2.7), the total covariance matrix in Eq. (5.9) can be rewritten in the following form,

$$
\begin{aligned}
C\left\{\hat{\boldsymbol{x}}_{p}\right\} & =\boldsymbol{I}-\boldsymbol{V}_{p} \tilde{\boldsymbol{V}}_{p}+\boldsymbol{V}_{p} \boldsymbol{\Lambda}_{p}^{-2} \tilde{\boldsymbol{V}}_{p} \\
& =\boldsymbol{V}_{0} \tilde{\boldsymbol{V}}_{0}+\boldsymbol{V}_{p} \boldsymbol{\Lambda}_{p}{ }^{-2} \tilde{\boldsymbol{V}}_{p},
\end{aligned}
$$

or

$$
C\left\{\hat{\boldsymbol{x}}_{p}\right\}=V \boldsymbol{D}_{p} \tilde{V}
$$

with

$$
V=\left[V_{p} \mid V_{0}\right], \quad D_{p}=\left[\begin{array}{c|c}
\Lambda_{p}^{-2} & O \\
\hline O & I_{m-p}
\end{array}\right]
$$

From Eq. (5.11), it is directly found that the noise in the data is active in the $V_{p^{-}}$ space, while the error in the initial guess is in the $V_{0}$-space. 
The solution produced by the generalized inverse operator $A^{\dagger}$ may be regarded as a straightforward extension of the classical least-squares solution. Actually, it minimizes the sum of squares of residuals. For simplicity, we refer to this solution as the "lesat-squares solution" hereafter. It is clear from the present error analysis that when the coefficient matrix $A$ has a very small eigenvalue, the estimation error becomes tremendously large, and so the least-squares solution losses any physical meaning.

\section{A Generalized Least-Squares Solution}

For many under-determined problems in geophysics, the smallest non-zero eigenvalue may be pretty small, and then the estimation error in the solution becomes unacceptably large. As pointed out by WIGGINs (1972), a sensible way to suppress the estimation error so that the solution has a physical meaning is to reconstruct the inverse operator out of the $q(\leq p)$ largest eigenvalues and the corresponding eigenvectors in the generalized inverse. One of the problems in such a "sharp cutoff approach" is how to determine a most appropriate "effective number," $q$, of degrees of freedom in data.

In order to determine the effective number $q$, for example, WIGGINs (1972) and also JACKSON (1972) set a threshold on the eigenvalues such that

$$
\sum_{j=1}^{q}\left(V_{i j} / \lambda_{j}\right)^{2} \leq{\sigma_{i}{ }^{2}}^{2} \quad(i=1, \cdots, m),
$$

where the left-hand side corresponds to the variance of estimate which results from the random noise in data, and the right-hand side to a maximum allowable variance determined a priori from our requirement for definiteness in the solution. In their formalisms, the effect of non-uniqueness in linear inversion due to poor resolution is not strictly taken into account, and so there remains some ambiguity in the determination of the effective number.

In the preceding section, we introduced the total covariance matrix of estimation error as a measure of uncertainties in the solution by combining the resolving error with the random error in the same unit. Then, in our formalism, the ambiguous problem of optimizing a trade-off between resolution and random error can be reduced to the difinite problem of minimizing the determinant of the covariance matrix. Here, it should be noted that the determinant of the covariance matrix gives a measure of the extent of a confidence region in the solution. The practical approach of the minimization is as follows:

We consider a linear inverse operator which is constructed out of the $k$ $(0 \leq k \leq p)$ largest eigenvalues and the corresponding eigenvectors in the generalized inverse. For the solution $\hat{\boldsymbol{x}}_{k}$ produced by this operator, the determinant of the covariance matrix is calculated by

$$
s_{k}^{2} \equiv\left|C\left\{\hat{\boldsymbol{x}}_{k}\right\}\right|=\prod_{i=1}^{k} 1 / \lambda_{i}^{2}
$$




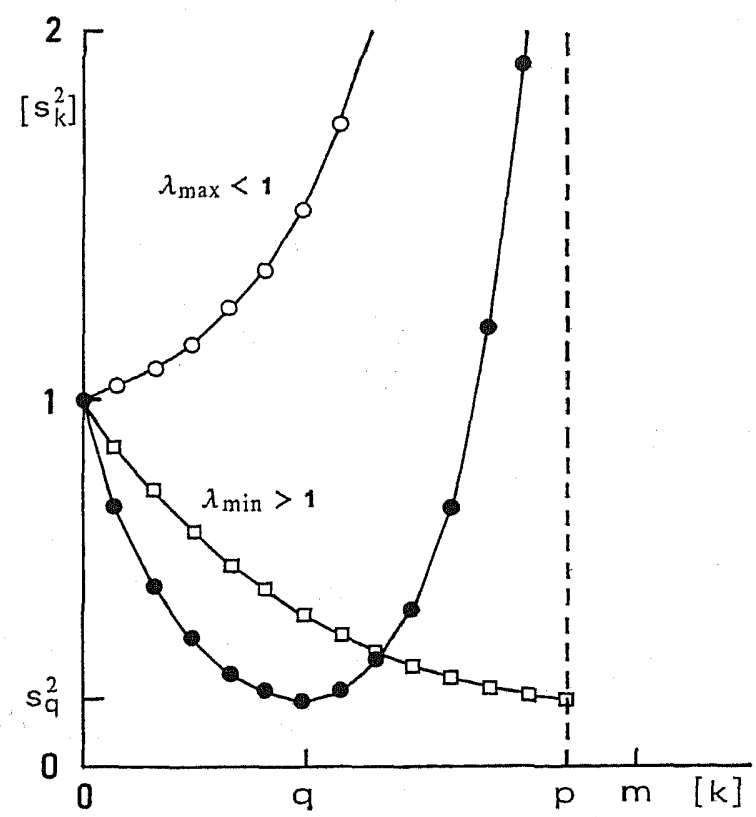

Fig. 1. Schematic diagram showing the variation of the determinant $s_{k}{ }^{2} \equiv\left|C\left\{\hat{x}_{k}\right\}\right|$ in Eq. (6.2) of the text with the number $k$ of the adopted eigenvalues. If $\lambda_{\max }=$ $\lambda_{1}<1$ (open circles), $s_{k}^{2}$ increases monotonously with $k$, and takes a minimum at $k=0$. If $\lambda_{\min }=\lambda_{p}<1$ (open squares), in contrast, $s_{k}^{2}$ decreases monotonously with $k$, and takes a minimum at $k=p$. In other cases (solid circles), $s_{k}^{2}$ has an extremal value at $k=q(0<q<p)$.

with

$$
C\{\hat{x} k\}=V D_{k} \tilde{V},
$$

where it should be noted that the determinant of the covariance matrix $C\left\{\hat{\boldsymbol{x}}_{k}\right\}$ is equivalent to the determinant of the matrix $D_{k}$ because of the orthogonality of $V$. Suppose that the $p$ positive eigenvalues are arranged in

$$
\lambda_{1}>\lambda_{2}>\cdots>\lambda_{q} \geq 1>\lambda_{q+1}>\cdots>\lambda_{p}>0 \text {. }
$$

Then, regarding $s_{k}{ }^{2}$ as a function of the parameter $k$, it varies with $k$ as shown in Fig. 1, and takes a minimum at $k=q(0<q<p)$, i.e.,

$$
\left(s_{t a}^{2}\right)_{\min }=s_{q}^{2}=\prod_{i=1}^{q} 1 / \lambda_{i}^{2} \leq 1
$$

This indicates that the minimization of the determinant of the covariance matrix is realized by merely truncating those eigenvalues which are smaller than unity.

It is quite natural to adopt the integer $q$ as the effective number of degrees of freedom in data, and then a best linear inverse operator is defined by

$$
\boldsymbol{A}_{q}^{\dagger}=\boldsymbol{V}_{q} \boldsymbol{\Lambda}_{q}^{-1} \tilde{U}_{q}
$$


which minimizes the determinant of the covariance matrix of estimation error within the framework of a generalized least-squares approach. The inverse operator $A_{q}^{\dagger}$ produces the correction vector,

$$
\Delta \hat{x}_{q}=A_{q}^{\dagger} \Delta y,
$$

and the solution vector and the corresponding covariance matrix are respectively written as

$$
\hat{\boldsymbol{x}}_{q}=A_{q}^{\dagger} A \boldsymbol{x}+\left(I-A_{q}^{\dagger} A\right) x^{0}+A_{q}^{\dagger} \boldsymbol{n}
$$

and

with

$$
C\left\{\hat{x}_{q}\right\}=V D_{q} \tilde{V}
$$

$$
D_{q}=\left[\begin{array}{c|c}
\Lambda_{q}^{-2} & O \\
\hline O & I_{m-q}
\end{array}\right]
$$

We refer to this solution as the "generalized least-squares solution" hereafter, to distinguish it from the least-squares solution in Eq. (5.8).

So far the problem has been treated in the standardized (dimensionless and statistically independent) system. All of the results obtained in the standardized system can be easily transformed back into the original system as

$$
\Delta \hat{X}_{q}=G^{-1} \Delta \hat{\boldsymbol{x}}_{q}, \quad \hat{X}_{q} \equiv X^{0}+\Delta \hat{X}_{q}=G^{-1} \hat{\boldsymbol{x}}_{q},
$$

and

$$
C\left\{\hat{\boldsymbol{X}}_{q}\right\}=\boldsymbol{G}^{-1} \boldsymbol{C}\left\{\hat{\boldsymbol{x}}_{q}\right\} \tilde{\boldsymbol{G}}^{-1} .
$$

From the definition, it is clear that the covariance matrix $C\left\{\hat{X}_{q}\right\}$ is symmetric and positive definite, and so the inverse of $C\left\{\hat{X}_{q}\right\}$ always exists. If we assume the Gaussian distribution both for the probable error $M$ in the initial guess and the random noise $N$ in the data, a confidence region for the solution is defined by the inequality,

$$
\left(\widetilde{X-\hat{X}_{q}}\right) C^{-1}\left\{\hat{X}_{q}\right\}\left(X-\hat{X}_{q}\right) \leq r^{2}
$$

which represents the region enclosed by a generalized second-order surface (hyperellipsoid) in the $m$-dimensional solution space. Note; if $\gamma=1$, Eq. (6.13) gives a confidence region corresponding to one standard error, if $\gamma=2$, that corresponding to two standard errors and so on.

From Eqs. (4.8) and (6.12), the $m$-dimensional volume $L^{m}\left(\hat{X}_{q}\right)$ of the confidence region is evaluated by

with

$$
L^{m}\left(\hat{X}_{q}\right)=\kappa\left|C\left\{\hat{X}_{q}\right\}\right|^{1 / 2}=\kappa\left|C\left\{\hat{x}_{q}\right\}\right|^{1 / 2}|C\{M\}|^{1 / 2}
$$

$$
\kappa=\gamma^{m} \times \begin{cases}\frac{\pi^{m / 2}}{(m / 2) !} & (m \text { is even }), \\ \frac{\pi^{(m-1) / 2} 2^{m}((m-1) / 2) !}{m !} & (m \text { is odd }) .\end{cases}
$$


Denoting the $m$-dimensional volume of the confidence region of the initial guess by $L^{m}\left(X^{0}\right)$, we obtain

$$
\boldsymbol{L}^{m}\left(\hat{X}_{q}\right)=S_{q} L^{m}\left(X^{0}\right) \leq L^{m}\left(X^{0}\right),
$$

where it is noticed that the factor $s_{q}$, which is defined in Eq. (6.5), represents a contraction rate of the confidence region. Namely, the generalized inverse process yields inevitably the contraction of the confidence region.

\section{Successive Iteration of Linear Inversion}

In quasi-linear inverse problems, the optimization of model parameters is made by the successive iteration of linear inversion. At the $k$-th $(k \geq 2)$ step of the successive iteration, the starting model $\boldsymbol{X}^{(k-1)}$ is given by the estimate $\hat{X}^{(k-1)}$ of one step earlier, and then the statistics of its probable error $M^{(k-1)}=\hat{X}^{(k-1)}-X$ is described by the corresponding covariance matrix $C\left\{\hat{\boldsymbol{X}}^{(k-1)}\right\}$; i.e.,

$$
\left.\begin{array}{l}
X^{(k-1)}=\hat{X}^{(k-1)} \\
C\left\{M^{(k-1)}\right\}=C\left\{\hat{X}^{(k-1)}\right\}
\end{array}\right\}
$$

Now the probable error $M^{(k-1)}$ in the starting model is not statistically independent of the random noise $N$ in data, and so the covariance matrix of estimate in the $k$-th step includes the cross terms of $M^{(k-1)}$ and $N$.

In the original system, for example, the estimate of the second step and the corresponding covariance matrix are written as follows;

$$
\hat{X}^{(2)}=X+\left(I-B_{(2)}^{\dagger} B_{(2)}\right)\left(\hat{X}^{(1)}-X\right)+B_{(2)}^{\dagger} N,
$$

and

$$
\begin{aligned}
C\left\{\hat{\boldsymbol{X}}^{(2)}\right\}= & \left.\left(I-\boldsymbol{B}_{(2)}^{\dagger} \boldsymbol{B}_{(2)}\right) C\left\{M^{(1)}\right\} \widehat{\left(\overline{\left.I-B_{(2)}^{\dagger} B_{(2)}\right)}\right.}\right) \\
& +B_{(2)}^{\dagger} C\{N\} \tilde{B}_{(2)}^{\dagger}+\left(I-B_{(2)}^{\dagger} B_{(2)}\right) B_{(1)}^{\dagger} C\{N\} \tilde{B}_{(2)}^{\dagger} \\
& +B_{(2)}^{\dagger} C\{N\} \tilde{B}_{(1)}^{\dagger}\left(\widetilde{I-B_{(2)}^{\dagger} B_{(2)}}\right)
\end{aligned}
$$

with

$$
\left.\begin{array}{ll}
\boldsymbol{B}_{(1)}=E^{-1} A G, & B_{(1)}^{\dagger}=G^{-1} A_{q_{1}}^{\dagger} E, \\
B_{(2)}=E^{-1} A^{\prime} G^{\prime}, & B_{(2)}^{\dagger}=G^{\prime-1} A_{q_{2}}^{\prime \dagger} E,
\end{array}\right\}
$$

and

$$
C\left\{M^{(1)}\right\}=C\left\{\hat{X}^{(1)}\right\}=\left(I-B_{(1)}^{\dagger} B_{(1)}\right) C\{M\}\left(\widetilde{I-B_{(1)}^{\dagger} B_{(1)}}\right)+B_{(1)}^{\dagger} C\{N\} \tilde{B}_{(1)}^{\dagger},
$$

where the matrices $\boldsymbol{E}$ and $\boldsymbol{G}$ are the same as those in Eq. (4.8), and $\boldsymbol{G}^{\prime}$ indicates the new weighting matrix defined by

$$
C^{-1}\left\{M^{(1)}\right\}=\tilde{G}^{\prime} \boldsymbol{G}^{\prime}
$$

In Eq. (7.3), the third and fourth terms correspond to the cross terms of $M^{(1)}$ and $N$.

Substituting the following expressions of $\boldsymbol{A}, \boldsymbol{A}_{q_{1}}^{\dagger}, \boldsymbol{A}^{\prime}, \boldsymbol{A}_{q_{2}}^{\prime \dagger}$, and $\boldsymbol{G}^{\prime}$, 


$$
\left.\begin{array}{ll}
A=U \Lambda \tilde{V}, & A_{q_{2}}^{\dagger}=V_{q_{1}} \Lambda_{q_{1}}^{-1} \tilde{U}_{q_{1}}, \\
A^{\prime}=U^{\prime} \Lambda^{\prime} \tilde{V}^{\prime}, & A_{q_{2}}^{\prime \dagger}=V_{q_{2}}^{\prime} \Lambda_{q_{2}}^{\prime-1} \tilde{U}_{q_{2}}^{\prime},
\end{array}\right\}
$$

and

$$
G^{\prime}=D_{q_{1}}^{-1 / 2} \tilde{V} G,
$$

into Eqs. (7.4) and (7.3), the covariance matrix can be rewritten in a simple form,

$$
C\left\{\hat{X}^{(2)}\right\}=G^{\prime-1} V^{\prime} D_{q_{2}}^{\prime} \tilde{V}^{\prime} \tilde{G}^{\prime-1},
$$

where

$$
D_{q_{2}}^{\prime}=\left[\begin{array}{l|l}
D_{11}^{\prime} & \frac{D_{12}^{\prime}}{D_{21}^{\prime}} \\
D_{22}^{\prime}
\end{array}\right]
$$

with

$$
\left.\begin{array}{l}
D_{11}^{\prime}=\Lambda_{q_{2}}^{\prime-2}, \quad D_{22}^{\prime}=I_{m-q_{2}}, \\
D_{12}^{\prime}=\tilde{D}_{21}^{\prime}=\left[\Lambda_{q_{2}}^{\prime-1} \tilde{U}_{q_{2}}^{\prime} U_{q_{1}} \mid O\right] V_{0}^{\prime}
\end{array}\right\}
$$

Then the determinant of the covariance matrix is evaluated as

$$
\left|C\left\{\hat{X}^{(2)}\right\}\right|=\left|D_{q_{2}}^{\prime}\right|\left|C\left\{M^{(1)}\right\}\right|
$$

with

$$
\left|D_{q_{2}}^{\prime}\right|=\left|D_{11}^{\prime}\right|\left|D_{22}^{\prime}-D_{21}^{\prime} D_{11}^{\prime-1} D_{12}^{\prime}\right| \text {. }
$$

Here, it should be noted that the matrix product $D_{21}^{\prime} D_{11}^{-1} D_{12}^{\prime}$ can be expressed by using the eigenvector matrices as

$$
D_{21}^{\prime} D_{11}^{\prime-1} D_{12}^{\prime}=\tilde{V}_{0}^{\prime}\left[\begin{array}{c|c}
\tilde{U}_{q_{1}} U_{q_{2}} \tilde{U}_{q_{2}} U_{q_{1}} & \boldsymbol{O} \\
\hline \boldsymbol{O} & \boldsymbol{O}_{m-q_{1}}
\end{array}\right] \boldsymbol{V}_{0}^{\prime} .
$$

When the problem is linear, the original coefficient matrix in the second step is exactly the same as that in the first step, and so the above process does not affect the solution at all. When the problem is nonlinear but weakly nonlinear, that is $\boldsymbol{B}_{(2)} \approx \boldsymbol{B}_{(1)}$, the singular value decomposition of the standardized coefficient matrix $\boldsymbol{A}^{\prime}$ in the second step becomes

$$
A^{\prime} \approx U \Lambda^{\prime} I
$$

Then, from the comparison of the above expression with Eq. (7.7), we obtain the approximate relations of $U^{\prime} \approx U$ and $V^{\prime} \approx I$. This indicates that the effects of the cross terms $\boldsymbol{D}_{12}^{\prime}$ and $\boldsymbol{D}_{21}^{\prime}$ are negligible, and so the matrix $\boldsymbol{D}_{q_{2}}^{\prime}$ in Eq. (7.9) may be replaced by the diagonal matrix $\boldsymbol{D}_{q_{2}}$, if the nonlinearity is weak.

When the nonlinearity is not so weak, it is required to evaluate exactly the covariance matrix of estimation error according to Eq. (7.9). This process will become much more complicated in the succeeding step of iterations. Recently, TARANTOLA and VALETTE (1982) have published an interesting paper, in which they attempted to give a generalization of the Franklin-Jackson's linear inverse formalism to nonlinear cases by defining the least-squares problem before lin- 
earization. Their approach is certainly an advisable way to overcome the difficulty in the usual iterative procedure. However, it should be noticed that there exists a hidden assumption of linearity in Tarantola and Valette's formalism, and so their solution has a physical meaning, if and only if $\left\|\hat{x}-x^{0}\right\|$ is so small that the "theoretical relationships" between data and model parameters may be regarded as linear around $x=x^{0}$.

Now let us consider the case where the problem is weakly nonlinear. In such a case, discarding the cross terms of the probable error in the starting model and the random noise in data, the generalized inversion at each step of the successive iteration is carried out in the same way as described in the preceding sections. Since the generalized inversion always reduces the confidence region in the solution, the iteration process of the inversion yields

$$
L_{\mathrm{min}}^{m} \leq \cdots \leq L^{m}\left(\hat{X}_{q_{k}}^{(k)}\right) \leq L^{m}\left(\hat{X}_{q_{k-1}(k-1)}\right) \leq \cdots \leq L^{m}\left(X^{0}\right)
$$

with

$$
L^{m}\left(\hat{X}_{q_{k}}^{\langle k\rangle}\right)=s_{q_{k}} L^{m}\left(\hat{X}_{q_{k-1}}^{\langle k-1\rangle}\right),
$$

where $L_{\min }^{m}$ indicates the $m$-dimensional volume of ultimate confidence region. In this iteration process, the effective number $q$ decreases step by step as the confidence region contracts, and then the contraction rate $s_{q}$ approaches to unity exponentially (Fig. 2). In fact, it can be easily understood that $q \approx m$ and $s_{q} \ll 1$

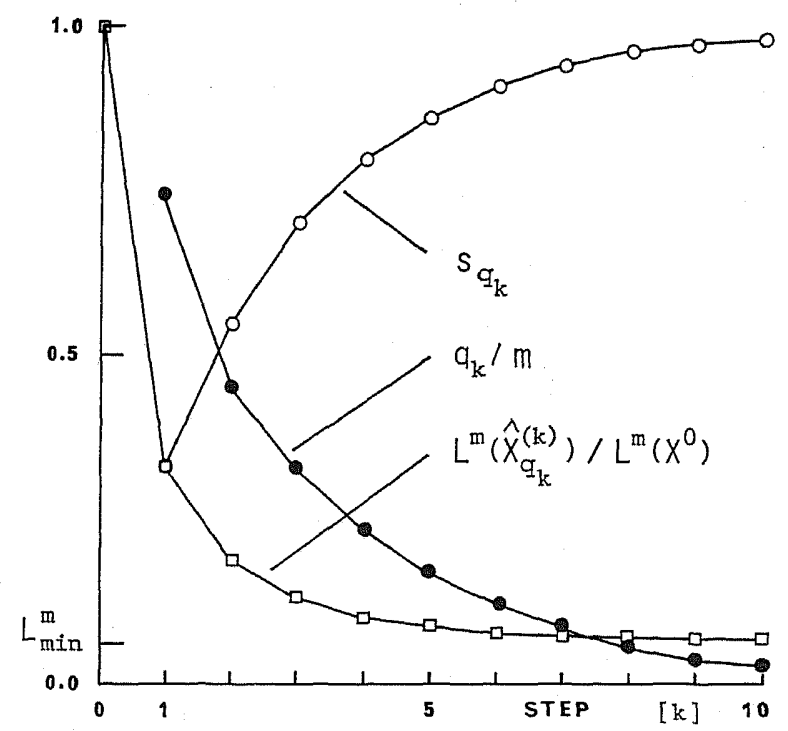

Fig. 2. Schematic diagram showing variations of the effective number $q_{k}$, the contraction rate $s_{q_{k}}$ and the $m$-dimensional volume $L^{m}\left(\hat{X}_{q_{k}}^{(k)}\right)$ of a confidence region in the successive iteration process of linear inversion. The normalizing factors, $m$ and $L^{m}\left(X^{0}\right)$, are the number of independent model parameters and the $m$-dimensional volume of the initial confidence region, respectively. 
if the confidence region for a starting model is extremely large, while $q \approx 0$ and $s_{q} \approx 1$ if the confidence region is very small. Accordingly, we can use the contraction rate $s_{q}$ to judge the convergence of the iteration process. Denoting a certain threshold by $\varepsilon$, the convergence criterion is given by

$$
1-s_{q}<\varepsilon
$$

\section{Comparison with a Minimum Variance Solution}

Recently, JACKSON (1979) demonstrated that an ill-posed linear problem can be always reduced to a well-posed linear problem by incorporating sufficient $a$ priori data into observed data, and then the problem may be solved by using the usual least-squares procedure. In this section, first of all, we give an outline of Jackson's formalism after some arrangements, and next, compare his result with our solutions.

Consider a set of the observed data equations and the a priori data equations in the standardized system, that is

$$
\left.\begin{array}{l}
y=A x+n \\
x^{0}=x+m
\end{array}\right\}
$$

with

$$
\boldsymbol{C}\{\boldsymbol{m}\}=I_{m}, \quad \boldsymbol{C}\{\boldsymbol{n}\}=I_{n},
$$

where $x^{0}$ is the initial guess obtained from a priori data, and $\boldsymbol{m}$ and $\boldsymbol{n}$ indicate the probable error in the initial guess and the random noise in the observed data, respectively. Rewriting Eq. (8.1) as

$$
\left.\begin{array}{l}
\Delta y=A \Delta x+n \\
0=\Delta x+m
\end{array}\right\}
$$

with

$$
\Delta x=x-x^{0}, \quad \Delta y=y-y^{0}, \quad y^{0}=A x^{0},
$$

and combining these two linear systems, we obtain a matrix equation to be solved as follows,

$$
\left[\frac{\Delta y}{0}\right]=\left[\frac{A}{I}\right] \Delta x+\left[\frac{n}{m}\right] .
$$

Since the above linear system is essentially over-determined, we may apply the usual least-squares procedure to solve it. Denoting the inverse operator by $H_{m}$, and the correction vector by $\Delta \hat{\boldsymbol{x}}_{m}$, the result is given by

$$
H_{m}=(\tilde{A} A+I)^{-1}\left[\tilde{A} \mid I_{m}\right],
$$

and

$$
\Delta \hat{x}_{m}=H_{m}\left[\frac{\Delta y}{0}\right]=(\tilde{A} A+I)^{-1} \tilde{A} \Delta y .
$$


Accordingly the solution vector $\hat{\boldsymbol{x}}_{m}$ and the covariance matrix of estimation error $\hat{\boldsymbol{x}}_{m}-\boldsymbol{x}$ in the solution are written as

$$
\hat{\boldsymbol{x}}_{m}=x+(\tilde{A} A+I)^{-1} m+(\tilde{A A}+I)^{-1} \tilde{A n}
$$

and

$$
C\left\{\hat{\boldsymbol{x}}_{m}\right\}=(\tilde{A} \boldsymbol{A}+I)^{-1} .
$$

In the special case of $x^{0}=0$, as pointed out by JACKSON (1979), the above solution is formally identical to the best linear solution of FRANKLIN (1970) which minimizes the total variances in Eq. (5.6). For this reason, we refer to Jackson's solution as the "minimum variance solution" hereafter.

Throughout this study, we have defined three different types of solutions to the linear inverse problem, such as the least-squares solution $\hat{x}_{p}$, the generalized least-squares solution $\hat{\boldsymbol{x}}_{q}$, and the minimum variance solution $\hat{\boldsymbol{x}}_{m}$. In the following part of this section, we try to clarify the physical meanings of these solutions by comparing them with each other.

In geophysical inverse problems, the solution $\hat{x}$ may be regarded as a good estimate of the true answer $\boldsymbol{x}$, if it satisfies the following criteria;

$$
C\{\hat{x}\} \approx 0, \quad r \approx 0,
$$

where $C\{\hat{x}\}$ is the covariance matrix of estimation error $\hat{x}-\boldsymbol{x}$ in the solutions, and $\boldsymbol{r}$ denotes the residual vector defined by

$$
r=\boldsymbol{y}-\boldsymbol{A} \hat{\boldsymbol{x}} \text {. }
$$

Decomposing the covariance matrix $C\{\hat{\boldsymbol{x}}\}$ and the residual vector $\boldsymbol{r}$ into standard form,

$$
\left.\begin{array}{l}
\boldsymbol{C}\{\hat{\boldsymbol{x}}\}=V \boldsymbol{V} \tilde{V} \\
\boldsymbol{r}=\tilde{U S} \tilde{U} \Delta y
\end{array}\right\}
$$

the matrices $D$ and $S$ are expressed as follows: for the least-squares solution $\hat{\boldsymbol{x}}_{p}$,

$$
D_{p}=\left[\begin{array}{c|c}
\Lambda_{p}^{-2} & \boldsymbol{O} \\
\hline \boldsymbol{O} & \boldsymbol{I}_{m-p}
\end{array}\right], \quad S_{p}=\left[\frac{\boldsymbol{O}_{p}}{\boldsymbol{O}} \mid \frac{\boldsymbol{O}}{\boldsymbol{I}_{n-p}}\right],
$$

for the generalized least-squares solution $\hat{\boldsymbol{x}}_{q}$,

$$
D_{q}=\left[\begin{array}{c|c}
\Lambda_{q}^{-2} & \boldsymbol{O} \\
\hline \boldsymbol{O} & \boldsymbol{I}_{m-q}
\end{array}\right], \quad S_{q}=\left[\frac{\boldsymbol{O}_{q}}{\boldsymbol{O}} \mid \frac{O}{\boldsymbol{I}_{n-q}}\right],
$$

and for the minimum variance solution $\hat{\boldsymbol{x}}_{m}$,

$$
\boldsymbol{D}_{m}=\left[\begin{array}{c|c}
\left(\boldsymbol{\Lambda}_{p}^{2}+\boldsymbol{I}_{p}\right)^{-1} & \boldsymbol{O} \\
\hline \boldsymbol{O} & \boldsymbol{I}_{m-p}
\end{array}\right], \quad \boldsymbol{S}_{m}=\left[\frac{\left(\boldsymbol{\Lambda}_{p}^{2}+\boldsymbol{I}_{p}\right)^{-1}}{\boldsymbol{O}} \mid \frac{\boldsymbol{O}}{\boldsymbol{I}_{n-p}}\right] .
$$

The physical meanings of the solutions are elucidated by examining the properties of the $D$-matrix and the $S$-matrix. In Fig. 3, values of the diagonal elements of these matrices are shown as discrete functions with respect to the number of an eigenvalue for two different over-constrained cases; (a) $q<p=m<n$ and (b) 

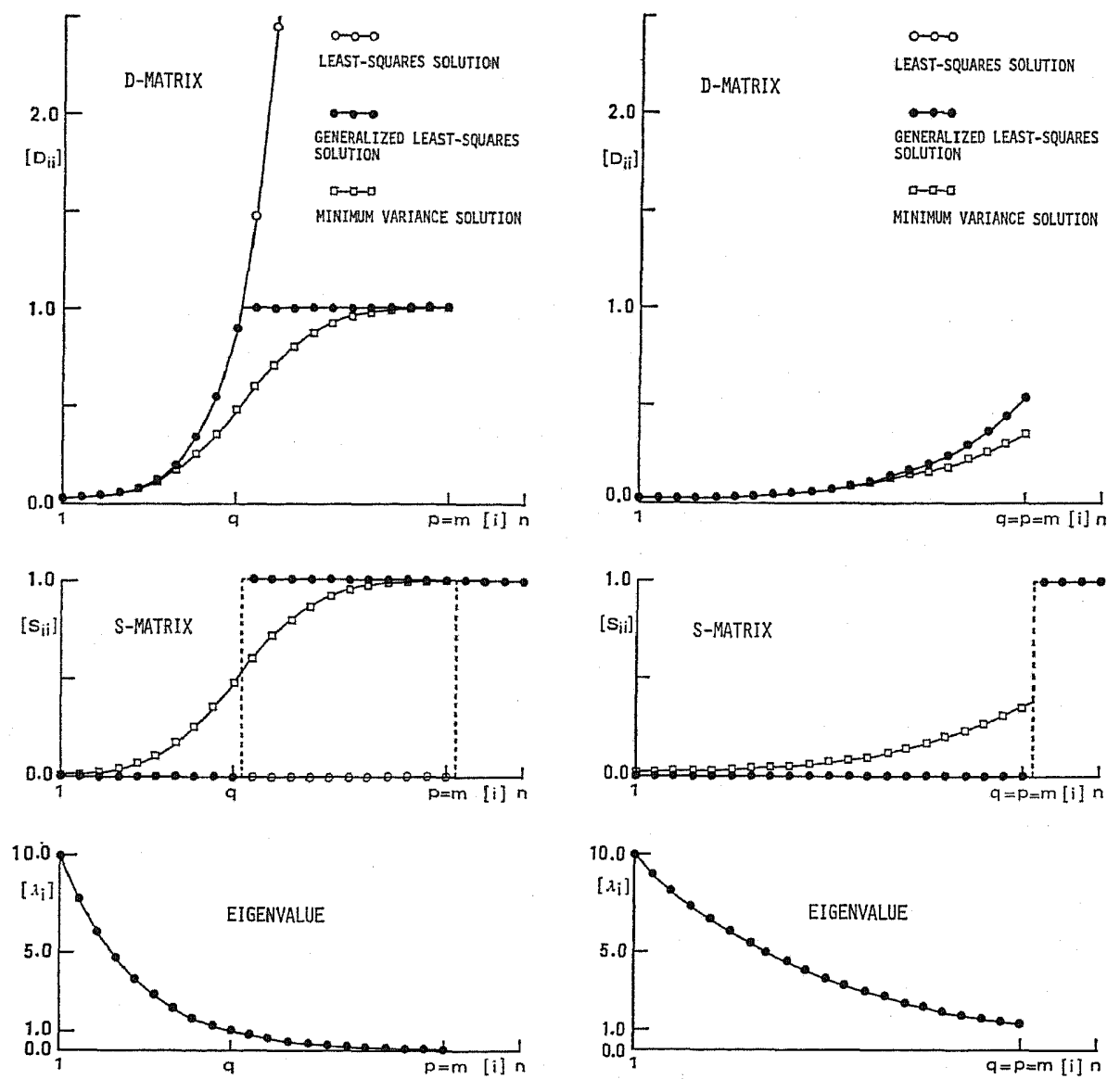

(a)

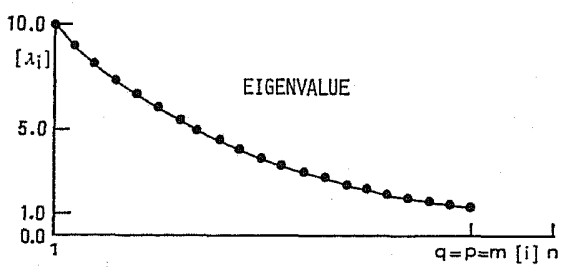

(b)

Fig. 3. Comparison of the fundamental properties of three different types of solutions for two representative cases; (a) $q<p=m<n$ and (b) $q=p=m<n$. In either case, the diagonal elements of $D$-matrix and $S$-matrix are respectively shown as the discrete functions of the number of an eigenvalue in the top and the middle of the figure. The distribution of eigenvalues adopted for the computation is given at the bottom of the figure.

$q=p=m<n$. In either case, the distribution of eigenvalues adopted for the computation is given at the bottom of the corresponding figure. As is seen from these figures, the generalized least-squares solution tends to the least-squares solution when the effective number $q$ approaches to $p(=m)$, and these solutions agree completely with each other if $q=p$. On the other hand, the minimum variance solution also tends to the least-squares solution when $q$ approaches $p$, but they never agree with each other even if $q=p$. Here, it should be noted that the effective number $q$ approaches $p$ as the $a$ priori constraints on model parameters become weak. When the a priori constraints are infinitely weak 
$(C\{M\} \rightarrow \infty \cdot I)$, the minimum variance solution agrees with the least-squares solution and also the generalized least-squares solution. However, such a situation seems to be unrealistic, since it is unlikely that we have no a priori information about model parameters in real geophysical problems.

\section{Discussions and Conclusions}

In geophysical inverse problems, we can guess a most plausible solution and the statistics of its probable error from a priori information about model parameters. When the problem is weakly nonlinear, starting from the most plausible solution, the optimization of the model parameters is made by the successive iteration of linear inversion. In under-determined cases, the solution depends inherently on the initial guess of the model parameters, and then estimation error in the solution includes not only the effect of random noise in data but also the effect of the probable error in the initial guess.

In this study, we treated the quasi-linear inverse problem with a priori information in terms of the singular value decomposition of rectangular matrices, and obtained a solution which minimizes the product of variances of estimation error within the framework of a generalized least-squares approach. This solution can be regarded as a natural extension of the classical least-squares solution, and provides a theoretical basis on the "sharp cutoff approach" of WIGGINS (1972) and also JACKsoN (1972) in their formalism. On the other hand, the minimum variance solution of FrANKLIN (1970) and also JACKSON (1979), which gives another generalization of the classical theory, provides a theoretical basis on the "tapered cutoff approach" of LeVENBerg (1944) and MARQUaRDT (1963, 1970). These two solutions, the generalized least-squares solution and the minimum variance solution, agree with each other in the particular case where the a priori constraints on model parameters are infinitely weak.

The authors are indebted to Professor David D. Jackson for critically reading the manuscript and offering helpful suggestions.

\section{REFERENCES}

BACKUS, G. and F. Gilbert, Numerical applications of a formalism for geophysical inverse problems, Geophys. J. R. Astron. Soc., 13, 247-276, 1967.

BACKus, G. and F. GILBERT, The resolving power of gross earth data, Geophys. J. R. Astron. Soc., 16, 169-205, 1968.

BACKUS, G. and F. GILBERT, Uniqueness in the inversion of inaccurate gross earth data, Philos. Trans. R. Soc. London, Ser. A, 266, 123-192, 1970.

ECKART, C. and G. Young, A principal axis transformation for non-Hermitian matrices, Am. Math. Soc. Bull., 45, 118-121, 1939.

FrankLIN, J. N., Well-posed stochastic extension of ill-posed linear problems, J. Math. Anal. Appl., 31, 682-716, 1970.

Hirata, N., T. Yamada, H. Shimamura, K. Suyehiro, and H. Inatani, Spatial distribution of 
microearthquakes beneath the Japan Trench from ocean bottom seismographic observations, Geophys. J. R. Astron. Soc., 1983 (in press).

JACKSON, D. D., Interpretation of inaccurate, insufficient and inconsistent data, Geophys. J. $R$. Astron. Soc., 28, 97-109, 1972.

JACKSON, D D., The use of a priori data to resolve non-uniqueness in linear inversion, Geophys. J. R. Astron. Soc., 57, 137-157, 1979.

KaulA, W. M., Theory of Satellite Geodesy, Chap. 5, Blaisdell, Waltham, Massachusetts, 1966.

LANCzos, C., Linear Differential Operators, Chap. 3, Van Nostrand, London, 1961.

LEVENBERG, K., A method for the solution of certain non-linear problems in least-squares, $Q$. Appl. Math., 2, 164-168, 1944.

MARQUARDT, D. W., An algorithm for least-squares estimation of nonlinear parameters, $J$. Soc. Ind. Appl. Math., 11, 431-441, 1963.

MARQUARDT, D. W., Generalized inverses, ridge regression, biased linear estimation, and nonlinear estimation, Technometrics, 12, 591-612, 1970.

MATSU'URA, M. and T. IWASAKI, Study on coseismic and postseismic crustal movements associated with the 1923 Kanto earthquake, Tectonophysics, Special Issue, 1983 (in press).

Penrose, R., A generalized inverse for matrices, Proc. Cambridge Philos. Soc., 51, 406-413, 1955.

TARANTOLA, A. and B. VALETTF, Generalized nonlinear inverse problems solved using the least squares criterion, Rev. Geophys. Space Phys., 20, 219-232, 1982.

WIGGINS, R. A., The general linear inverse problem: Implication of surface waves and free oscillations for earth structure, Rev. Geophys. Space Phys., 10, 251-285, 1972. 